\title{
Metabolic engineering of Saccharomyces cerevisiae for high-level production of gastrodin from glucose
}

Hua Yin ${ }^{1,2}$, Tiandong Hu ${ }^{1,2}$, Yibin Zhuang ${ }^{1,2}$ and Tao Liu ${ }^{1,2^{*}}$

\begin{abstract}
Background: The natural phenolic glycoside gastrodin is the major bioactive ingredient in the well-known Chinese herb Tianma and is widely used as a neuroprotective medicine in the clinic. Microbial production from sustainable resources is a promising method to replace plant extraction and chemical synthesis which were currently used in industrial gastrodin production. Saccharomyces cerevisiae is considered as an attractive host to produce natural plant products used in the food and pharmaceutical fields. In this work, we intended to explore the potential of S. cerevisiae as the host for high-level production of gastrodin from glucose.

Results: Here, we first identified the plant-derived glucosyltransferase AsUGT to convert 4-hydroxybenzyl alcohol to gastrodin with high catalytic efficiency in yeast. Then, we engineered de novo production of gastrodin by overexpressing codon-optimized AsUGT $T^{s y n}$, the carboxylic acid reductase gene $C A R^{s y n}$ from Nocardia species, the phosphopantetheinyl transferase gene PPTcg-1 $1^{\text {syn }}$ from Corynebacterium glutamicum, the chorismate pyruvate-lyase gene UbiC${ }^{s y n}$ from Escherichia coli, and the mutant ARO4 ${ }^{K 229 L}$. Finally, we achieved an improved product titer by a chromosomal multiple-copy integration strategy and enhancement of metabolic flux toward the aglycon 4-hydroxybenzyl alcohol. The best optimized strain produced $2.1 \mathrm{~g} / \mathrm{L}$ gastrodin in mineral medium with glucose as the sole carbon source by flask fermentation, which was 175 times higher than that of the original gastrodin-producing strain.
\end{abstract}

Conclusions: The de novo high-level production of gastrodin was first achieved. Instead of chemical synthesis or plants extraction, our work provides an alternative strategy for the industrial production of gastrodin by microbial fermentation from a sustainable resource.

Keywords: Saccharomyces cerevisiae, Gastrodin, AsUGT, Phenolic glycoside, Chromosomal integration, Metabolic engineering

\section{Background}

The phenolic glycoside gastrodin is the major bioactive ingredient in the famous Chinese herb Tianma (the tuber of Gastrodia elata Blume), which has been widely used to treat nervous system diseases, such as ischemic stroke, epilepsy, dizziness, and dementia, for centuries in China

*Correspondence: liu_t@tib.cas.cn

${ }^{1}$ Tianjin Institute of Industrial Biotechnology, Chinese Academy of Sciences, Tianjin 300308, China

Full list of author information is available at the end of the article
$[1,2]$. In addition to its neuroprotective effects [3], gastrodin has been proven to show memory improvement [4], anti-inflammation, and antioxidant effects [5, 6]. To date, gastrodin is produced mainly by chemical synthesis or direct extraction from Tianma, although different processing methods have also been explored and applied $[7,8]$. However, total chemical synthesis is a complicated process and leads to severe environmental pollution due to the use of toxic phenols, phosphate and bromide. Direct extraction from Tianma is costly and time consuming because of the low gastrodin content in Tianma

(C) The Author(s) 2020. This article is licensed under a Creative Commons Attribution 4.0 International License, which permits use, sharing, adaptation, distribution and reproduction in any medium or format, as long as you give appropriate credit to the original author(s) and the source, provide a link to the Creative Commons licence, and indicate if changes were made. The images or other third party material in this article are included in the article's Creative Commons licence, unless indicated otherwise in a credit line to the material. If material is not included in the article's Creative Commons licence and your intended use is not permitted by statutory regulation or exceeds the permitted use, you will need to obtain permission directly from the copyright holder. To view a copy of this licence, visit http://creativeco mmons.org/licenses/by/4.0/. The Creative Commons Public Domain Dedication waiver (http://creativecommons.org/publicdomain/ zero/1.0/) applies to the data made available in this article, unless otherwise stated in a credit line to the data. 
(below 0.7\%) [8]. Meanwhile, wild resources of Tianma are scarce, and its artificial cultivation is relatively complicated due to the nature of its symbiotic relationship with two compatible mycorrhizal fungi during seed germination and vegetative growth [9]. The success of heterologous production of plant-derived natural products in microorganisms provides an alternative strategy for producing gastrodin in an economical and environmentally friendly manner [10].

The native biosynthetic pathway of gastrodin in plant has still not been documented. In our previous work [11], we first achieved microbial biosynthesis of gastrodin by constructing an artificial pathway in Escherichia coli, beginning with the conversion of chorismate to 4-hydroxybenzoic acid (4-HBA) by chorismate pyruvate-lyase (UbiC). Then, 4-HBA was transformed to 4-hydroxybenzyl aldehyde by carboxylic acid reductase (CAR), which is activated into its holo form by a phosphopantetheinyl transferase (sfp). The 4-hydroxybenzyl aldehyde was transformed to 4-hydroxybenzl alcohol by endogenous alcoho9l dehydrogenases (ADHs). Finally, 4-hydroxybenzl alcohol was converted to gastrodin by the glycosyltransferase UGT73B6 from Rhodiola. With further rational engineering of the glycosyltransferase, the resulting E. coli strain produces approximately $0.5 \mathrm{~g} / \mathrm{L}$ gastrodin. Our previous results strongly suggest that the activity of UGTs is critical for the improvement of gastrodin biosynthesis. Unfortunately, the native glucosyltransferase for gastrodin biosynthesis is unknown. Tsai et al. [9] reported that a unigene encoding glycosyltransferase might participate in the gastrodin native biosynthetic pathway. However, its sequence has not yet been published. To date, UGT73B6 from Rhodiola is the only glycosyltransferase that has been characterized to be functional in gastrodin synthesis.

As common microbial platforms, E. coli and Saccharomyces cerevisiae have been engineered to produce aromatic amino acid-derived (AAA-derived), chorismate-derived or shikimate-derived products [12]. As in $E$. coli, some successful strategies have also been used in yeast to enhance the production of these products to gram levels per liter [13, 14]. Liu et al. [15] constructed an $S$. cerevisiae platform strain with an AAA-derived $p$-coumaric acid yield of more than $10 \mathrm{~g} / \mathrm{L}$ by rewiring carbon metabolism, achieving appropriate levels for large-scale production. Food-grade $S$. cerevisiae is considered an attractive host due to its superior capability of expressing enzymes requiring posttranslational modification, safety, robust fermentation and resistance to phage infection [16].

In this work, we explored the potential of S. cerevisiae as the host to produce gastrodin. We first identified a glucosyltransferase with high catalytic efficiency for gastrodin synthesis and constructed a gastrodinproducing yeast strain by pathway engineering. By a chromosomal integration strategy and metabolic flux enhancement toward the 4-hydroxybenzyl alcohol, we achieved high-level production of gastrodin. Engineered yeast strains could serve as the basis for creating an alternative method to produce gastrodin, instead of extraction from plant sources or chemical synthesis in the future.

\section{Results and discussion \\ The glucosyltransferase AsUGT catalyzed gastrodin biosynthesis with high efficiency in yeast}

To achieve heterologous biosynthesis of gastrodin in yeast, it is necessary to identify a compatible glucosyltransferase that recognizes 4-hydroxybenzyl alcohol as a substrate with high efficiency. It was reported that the glucosyltransferase AS from $R$. serpentina could accept phenols as substrates [17]. Based on the structural similarity between phenols and 4-hydroxybenzyl alcohol, we hypothesized that AS might be able to catalyze the glucosylation of 4-hydroxybenzyl alcohol. To test this hypothesis, we overexpressed a codon-optimized AS (named $A s U G T^{s y n}$ in this work) in yeast and carried out feeding experiments with 4-hydroxybenzyl alcohol as the substrate. HPLC analyses of the metabolites in the fermentation broth supernatants produced by the engineered strain displayed a new peak at a retention time of $6.3 \mathrm{~min}$ (Fig. 1a). HPLC-MS analysis showed that the new compound had a molecular ion at $\mathrm{m} / z 304.1419$ $\left(\left[\mathrm{M}+\mathrm{NH}_{4}\right]^{+}\right)$, which was identical to that of gastrodin (Fig. 1b). Approximately $0.5 \mathrm{mM}$ gastrodin was produced within $24 \mathrm{~h}$, and the substrate conversion rate was $25 \%$. Within $48 \mathrm{~h}$, the substrate conversion rate reached up to $55 \%$, with approximately $1.1 \mathrm{mM}$ gastrodin produced.

Our previous study demonstrated that the glucosyltransferase UGT73B6 ${ }^{\mathrm{FS}}$, a mutant of UGT73B6, showed high catalytic efficiency in the conversion of 4-hydroxybenzyl alcohol to gastrodin in E. coli [11]. To compare enzymatic efficiency between codon-optimized AsUGT ${ }^{s y n}$ and $U G T 73 B 6^{F S y}$ in yeast, ScTEF1 promoter-driven $U G T 73 B 6^{F S y}$ was also introduced into $S$. cerevisiae BY4742. The resulting strain $4742-U G T 73 B 6^{\mathrm{FS}}$ was cultivated under the same conditions. As shown in Fig. 1c, strain 4742-UGT73B6 ${ }^{\mathrm{FSy}}$ produced only trace amounts of gastrodin. The substrate conversion rate in strain 4742AsUGT $^{\text {syn }}$ was 33 times higher than that in strain 4742 $\mathrm{UGT} 3 \mathrm{BB}^{\mathrm{FSy}}$ within $24 \mathrm{~h}$. Taken together, these results clearly showed that AsUGT exhibited a higher catalytic efficiency than UGT73B6 ${ }^{\mathrm{FS}}$ in yeast. Thus, AsUGT was used for heterologous gastrodin biosynthesis in subsequent experiments. 


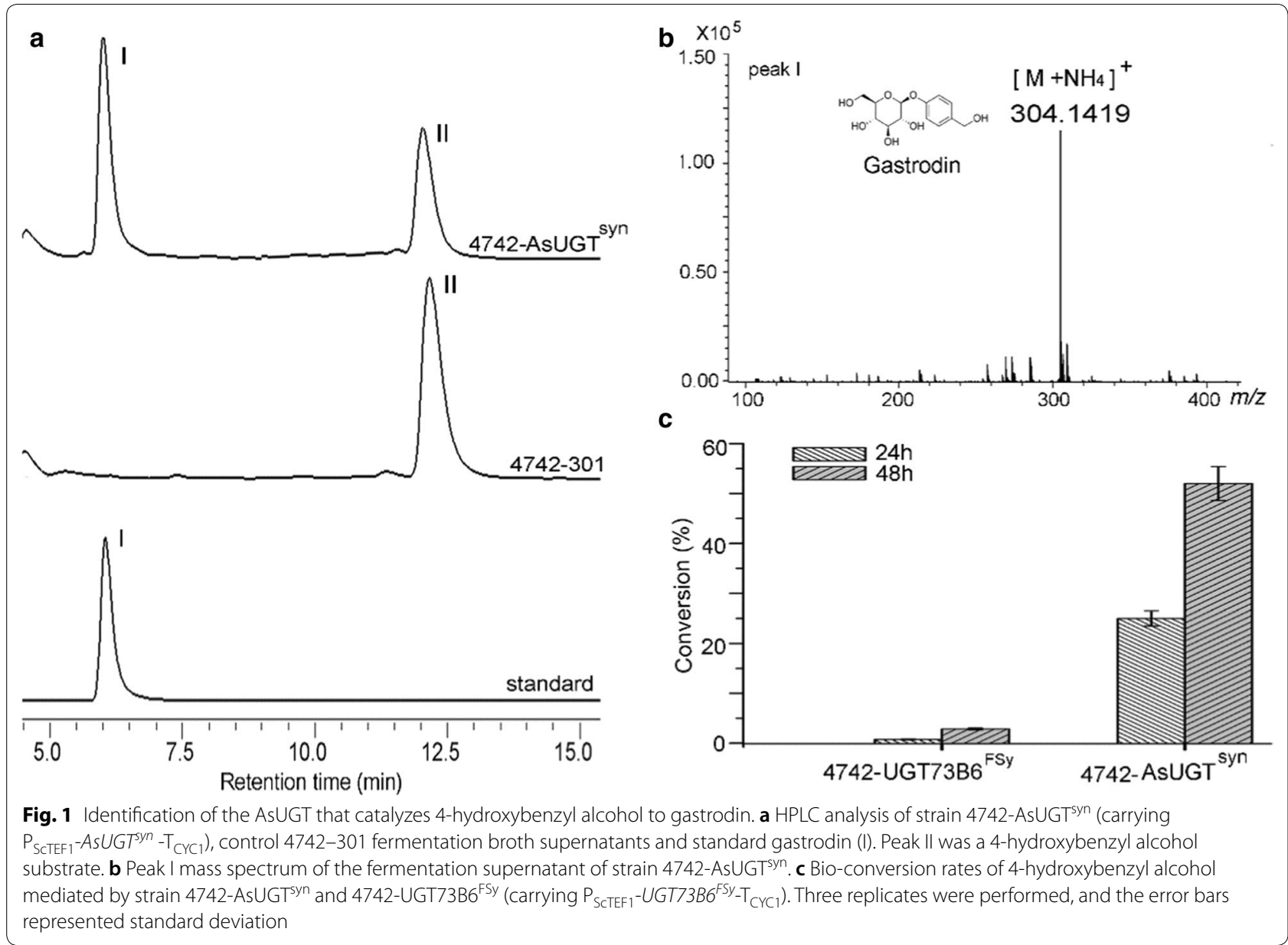

\section{De novo biosynthesis of gastrodin from glucose was achieved in S. cerevisiae}

As shown in our previous study, the artificial gastrodin biosynthesis pathway was extended from chorismate [11]. In bacteria, chorismate lyase (UbiC) usually catalyzed chorismate to synthesize 4-HBA and the amounts of 4-HBA always low in cultures [18]. In $S$. cerevisiae, a homolog of $\mathrm{UbiC}$ is missing, and the biosynthetic pathway of 4-HBA is unclear [19]. There was no detectable 4-HBA accumulation in $S$. cerevisiae [20]. Overexpression of the feedback inhibition resistance gene $A R O 4^{K 229 L}$ is usually used to improve the chorismate pathway metabolic flux [14]. In this work, we intended to overexpress $A R O 4^{K 229 L}$ and UbiC from E. coli to preliminarily produce 4-HBA from chorismate in yeast. Coexpression of CAR from the Nocardia genus and PPTcg-1 from C. glutamicum could effectively reduce protocatechuic acid to protocatechuic aldehyde in S. cerevisiae [21]. We tried to overexpress CAR from the Nocardia genus and PPTcg-1 from $C$. glutamicum in yeast to realize the reduction of 4-HBA to aldehyde. The subsequent reduction of aldehyde to 4-hydroxybenzylalcohol might be achieved by endogenous alcohol dehydrogenase (ADHs) in yeast. The de novo biosynthetic pathway of gastrodin in S. cerevisiae is shown in Fig. 2. We constructed $2 \mu$-based plasmids carrying strong constitutive promoters to realize target gene overexpression. The plasmid pCf302-CP and the plasmid pCf301-AUA were introduced into S. cerevisiae BY4742, and the resulting strain was named 4742-pGS. We observed two clear new compound peaks at retention times of $6.3 \mathrm{~min}$ (peak I) and $12.6 \mathrm{~min}$ (peak II) in the HPLC spectrum of the fermentation broth supernatant of 4742-pGS compared to that of control 4742-pCf (Fig. 3a). LC-MS analysis showed that peak I had a molecular ion at $m / z$ $304.1419\left(\left[\mathrm{M}+\mathrm{NH}_{4}\right]^{+}\right)$, which was identical to that of standard gastrodin (Fig. 3b). Peak II had a molecular ion at $m / z 107.0515\left(\left[\mathrm{M}-\mathrm{H}_{2} \mathrm{O}+\mathrm{H}\right]^{+}\right)$, which was identical to that of standard 4-hydroxybenzyl alcohol (Fig. 3c). The results clearly showed that gastrodin and 4-hydroxybenzylalcohol were produced in strain 4742-pGS, demonstrating that the pathway genes $C A R$ syn, $P P T c g-1^{\text {syn }}$ and $A s U G T^{s y n}$ were functional in yeast. 

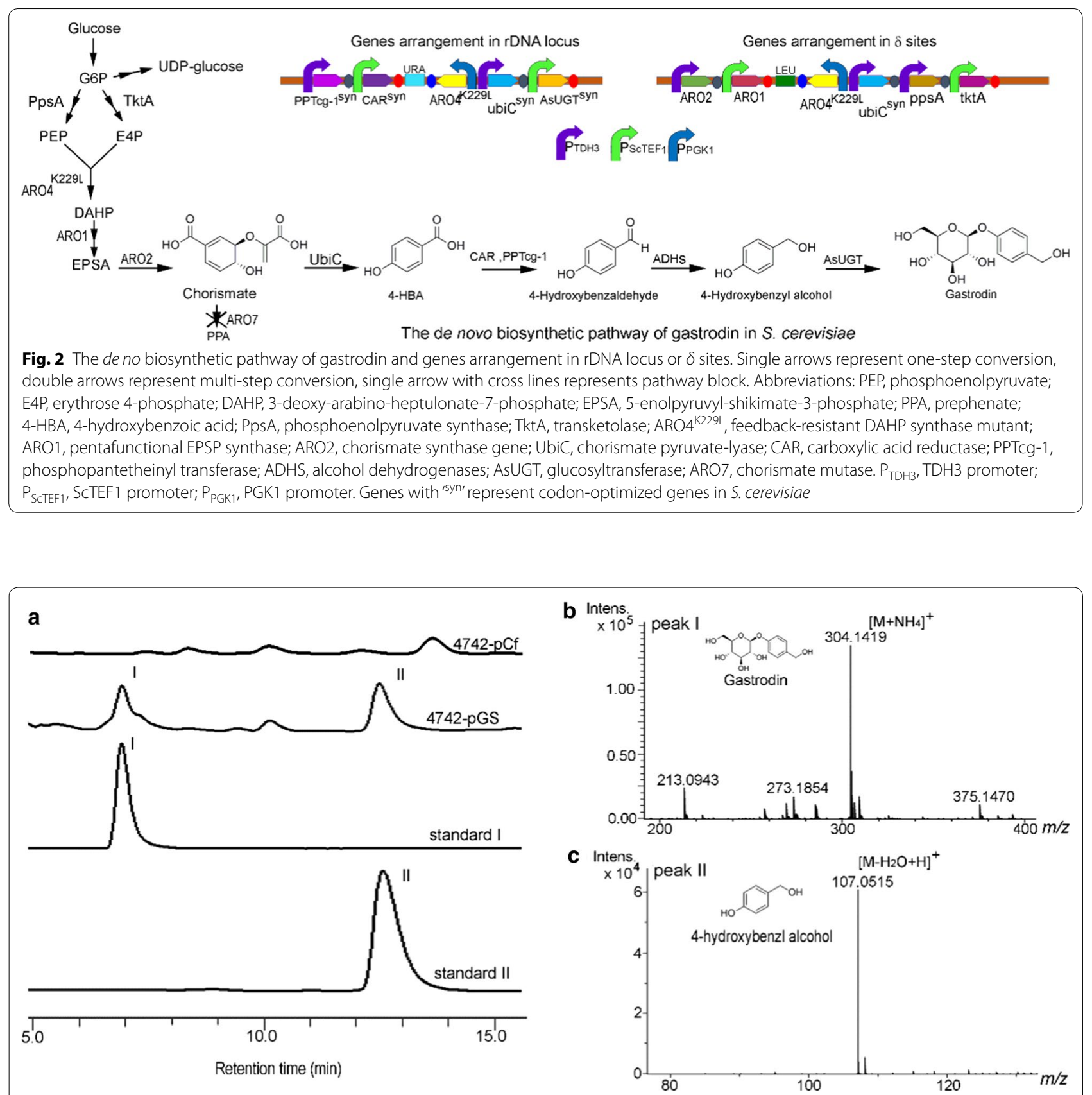

Fig. 3 De novo biosynthesis of gastrodin in strain 4742-pGS. a HPLC analysis of standard gastrodin (I), standard 4-hydroxybenzyl alcohol (II) and products in the fermentation supernatant of the recombinant strains 4742-pGS (S. cerevisiae BY4742 with plasmid pCf302-CP and pCf301-AUA, carrying $C A R^{s y n}, P P T c g-7^{\text {syn }}, A R O 4^{K 229 L}$, ubi $C^{\text {yyn }}$ and $A s U G T^{\text {syn }}$ ) and 4742-pCf (control). b Mass analysis of peak I from the fermentation supernatant of strain 4742-pGS. c Mass analysis of peak II from the fermentation supernatant of strain 4742-pGS

4-Hydroxybenzylalcohol was synthesized from 4-HBA by CAR reduction, and subsequently, a portion of the 4-hydroxybenzylalcohol was glycosylated to gastrodin by AsUGT in S. cerevisiae BY4742. The overexpression of $A R O 4^{K 229 L}$ and E. coli UbiC might improve the production of 4-HBA from chorismate.

\section{Gastrodin production was improved by chromosomal} integration of biosynthetic pathway genes in the rDNA locus

Stable integration and robust expression of foreign genes is critical for the success of heterologous biosynthesis of valuable compounds. It is challenging to stably express 
multiple genes in S. cerevisiae with the generally used CEN ori-based and $2 \mu \mathrm{m}$ ori-based plasmids as tools [22, 23]. Moreover, plasmid-based pathway gene expression could result in growth and genetic instability [24]. Chromosomal integration is an ideal method for introducing heterologous genes to achieve robust expression because of its structural stability [25]. The rDNA encompasses approximately 140 copies of tandem repeats on chromosome XII [26], and $\delta$ sequences exist in over 100 copies on chromosome XV [27]. The rDNA locus or $\delta$ sites are ideal for generating multicopy integration. Some genes associated with natural products, such as ginsenosides [28], glycyrrhetinic acid [29], and salidroside [30], have been successfully integrated into the rDNA locus or $\delta$ sites in yeast to achieve multiple-copy and stable expression by different biotechnological methods.

We aimed to simultaneously integrate $C A R^{s y n}, P P T c g$ $1^{s y n}, A s U G T^{s y n}, u b i C^{s y n}$ and $A R O 4^{K 229 L}$ into the yeast chromosomal rDNA locus in the expected order by onestep transformation. The arrangement of these genes in the rDNA locus is shown in Fig. 2. In S. cerevisiae, the knockout of chorismate mutase (ARO7) could block chorismate flux to prephenate and activate the shikimate pathway [20]. Accordingly, we constructed the aro7s mutant in S. cerevisiae BY4742 as the parental strain. First, we PCR-amplified two DNA fragments containing the five target genes and URA3 auxotrophic marker from constructed plasmids in the work. Together with the two fragments rDNA-up and rDNA-down, these DNA fragments (approximately $14 \mathrm{~kb}$ ) were simultaneously transformed into the strain S. cerevisiae aro7 $\Delta$ by a one-step multiple-fragment yeast transformation protocol. The assembly order and integration of the DNA fragments into the rDNA locus were confirmed by diagnostic PCR and sequencing. Positive colonies were selected and cultured to determine gastrodin production, as shown in Additional file 1: Figure S1. The resulting strain with the highest concentration of gastrodin was named rGS3.

Another, we introduced the plasmids pCf302-CP and pCf301-AUA carrying $C A R^{s y n}, P P T c g-1^{s y n}, A s U G T$ ${ }^{s y n}, u b i C^{s y n}$ and $A R O 4^{K 229 L}$ into S. cerevisiae aro $7 \Delta$. The generated plasmid-based strain was named $\Delta 7$-pGS. Gastrodin production in rGS3 and $\Delta 7$-pGS was measured under the same fermentation conditions. Based on the production curves (Fig. 4a), gastrodin production in strain $\Delta 7$-pGS reached approximately $120 \mathrm{mg} / \mathrm{L}$ and was nearly stable after four days of fermentation. The gastrodin titer of strain rGS3 reached approximately $420 \mathrm{mg} / \mathrm{L}$ after 6 days of culture, which was 3.8 times higher than that of the plasmid-based strain $\Delta 7$-pGS. HPLC spectra are shown in Fig. 4b. The growth curves showed that there was a growth decline after two days of cultivation of $\Delta 7$-pGS, and the $\mathrm{OD}_{600}$ of strain rGS3 was $30 \%$ higher than that of $\Delta 7$-pGS at the end of fermentation (Fig. 4a). These results confirmed that the gene chromosomal integration strategy effectively improved gastrodin production and brought growth recovery compared to plasmid-based pathway gene expression.

\section{Further improving the precursor supply increased gastrodin production}

Enhancement of the precursor supply is a classic and effective strategy to improve final compound production. The initiation of chorismate biosynthesis requires two

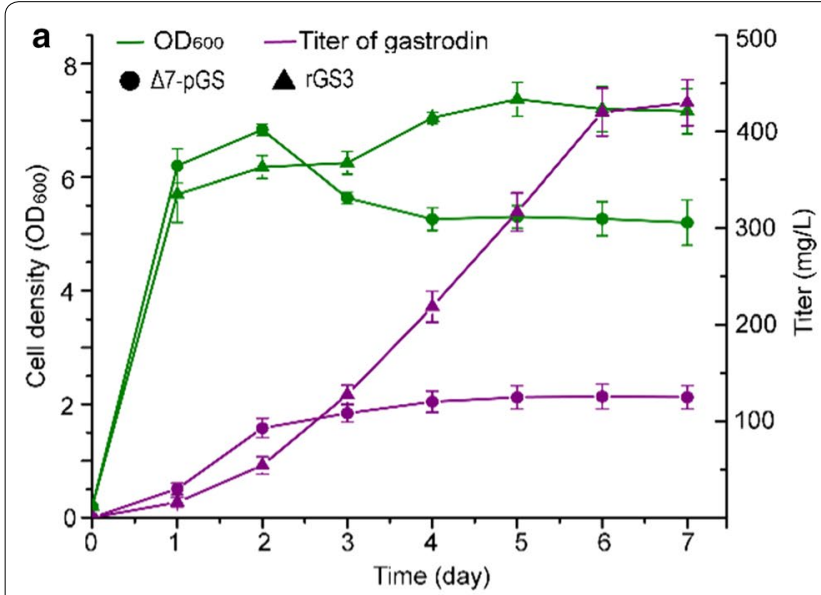

b

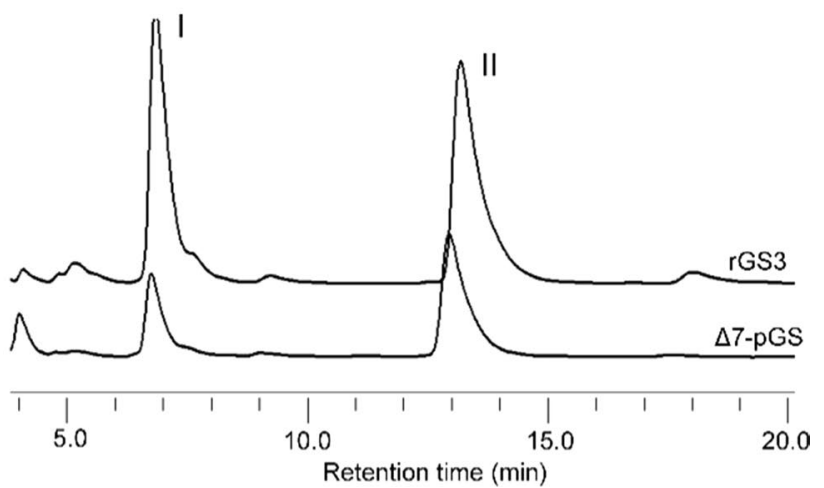

Fig. 4 The differential comparison of gastrodin production and growth of strains rGS3 and $\triangle 7-p G S$. a Time profile of gastrodin production and cell density of strains rGS3 (with pathway genes integrated into rDNA locus) and $\triangle 7-p G S$ (with pathway genes expressed in plasmids) by flask fermentation. b HPLC analysis of gastrodin (I) and 4-hydroxybenzyl alcohol (II) in the fermentation supernatant of strains 7-pGS and rGS3 over 6 days of culture. Three replicates were performed, and the error bars represented standard deviation 
precursors: erythrose-4-phosphate (E4P) derived from the pentose phosphate pathway and phosphoenolpyruvate (PEP) derived from glycolysis. In S. cerevisiae, E4P and PEP were not abundant and were pathway bottlenecks based on the results of carbon tracing and ${ }^{13} \mathrm{C}$ metabolic flux analysis [31]. Many studies have revealed that the overexpression of transketolase (TktA) and phosphoenolpyruvate synthase (PpsA) effectively raised the intracellular pool of E4P and PEP [32, 33]. In yeast, the 3-deoxyD-arabino-heptulosonate-7-phosphate (DAHP) synthase (ARO4/ARO3) catalyzes the first step to condense PEP and E4P to form DAHP. The pentafunctional enzyme (ARO1) catalyzes five subsequent reactions from DAHP to 5-enolpyruvyl-shikimate-3-phosphate (EPSP). Chorismate synthase $(A R O 2)$ catalyzes the final conversion of EPSP to chorismate. Previous reports showed that co-overexpression of $A R O 2, A R O 1$ and ARO4 (or ARO3) could increase AAA-derived aromatic chemical production [15].

Given that a deficient supply of precursors might be the cause of the low amount of gastrodin, we intended to further enhance the carbon flux into 4-HBA precursor accumulation by combining the overexpression of ppsA, $t k t A$, $A R O 1$, and $A R O 2$ using the multiple-copy integration strategy. First, we PCR-amplified three large DNA fragments containing ppsA, tktA, ARO1, ARO2, the mutated $A R O 4^{K 229 L}$ and $u b i C^{s y n}$ from constructed plasmids in the work. Together with the two fragments $\delta$ DNA-up and $\delta$ DNA-down, these DNA fragments (approximately $21 \mathrm{~kb}$ ) were simultaneously transformed into the strain S. cerevisiae aro7s by a one-step multiple-fragment yeast transformation protocol. The arrangement of these genes in $\delta$ sites is shown in Fig. 2. The assembly order and integration of the DNA fragments into the $\delta$ sites were confirmed by diagnostic PCR and sequencing. Positive colonies were selected and cultured. HPLC analysis of the culture of these colonies showed that there was a clear peak I at a retention time of $28.5 \mathrm{~min}$, similar to the 4-HBA standard, while peak I was absent in S. cerevisiae aro7 $\triangle$ (Fig. 5a). We collected the fraction of peak I for LC-MS in negative ion mode in a methanol-water (containing $20 \mathrm{mM} \mathrm{NH} \mathrm{m}_{4} \mathrm{Ac}$ ) gradient system. LC-MS analysis showed that peak I had a molecular ion at $\mathrm{m} / \mathrm{z}$ $137.0189\left([\mathrm{M}-\mathrm{H}]^{-}\right)$, which was identical to that of standard 4-HBA (Fig. 5b), further confirming 4-HBA overproduction. Production of 4-HBA in some positive colonies was shown in Additional file 1: Figure S2. The colony with the highest 4-HBA production was named $\triangle 7$-HBA. Another, consistent with a previous report [20], there was no detectable 4-HBA accumulation in S. cerevisiae aro $7 \triangle$. These results demonstrated that the combined overexpression of ARO1, ARO2, mutated $A R O 4^{K 229 L}$, ppsA, $t k t A$ and $u b i C^{s y n}$ effectively enhanced metabolic flux toward 4-HBA biosynthesis in yeast.

Subsequently, these five DNA fragments were transformed into S. cerevisiae rGS3. Gastrodin production in the positive colonies was measured (Additional file 1: Figure S3), and the colony with the highest gastrodin production was named rGS-HBA. To evaluate the product titers and cell density of the engineered strains, we carried out fermentation in mineral medium with glucose as the sole carbon source by a $250-\mathrm{mL}$ flask fermentation. The titers of gastrodin, 4-hydroxybenzyl alcohol and 4-HBA were determined after 6 days of fermentation. As shown in Fig. 6, the original gastrodin-producing strain 4742-pGS had $12 \mathrm{mg} / \mathrm{L}$ gastrodin, accumulated $8 \mathrm{mg} / \mathrm{L}$ 4-hydroxybenzyl alcohol and $120 \mathrm{mg} / \mathrm{L}$ 4-HBA. In strain 4742-pGS, the accumulation of 4-HBA showed that the overexpression of $\mathrm{ARO} 4{ }^{\mathrm{K} 229 \mathrm{~L}}$ and $E$. coli $\mathrm{UbiC}$ indeed enhanced 4-HBA production. Compared to 4742-pGS,
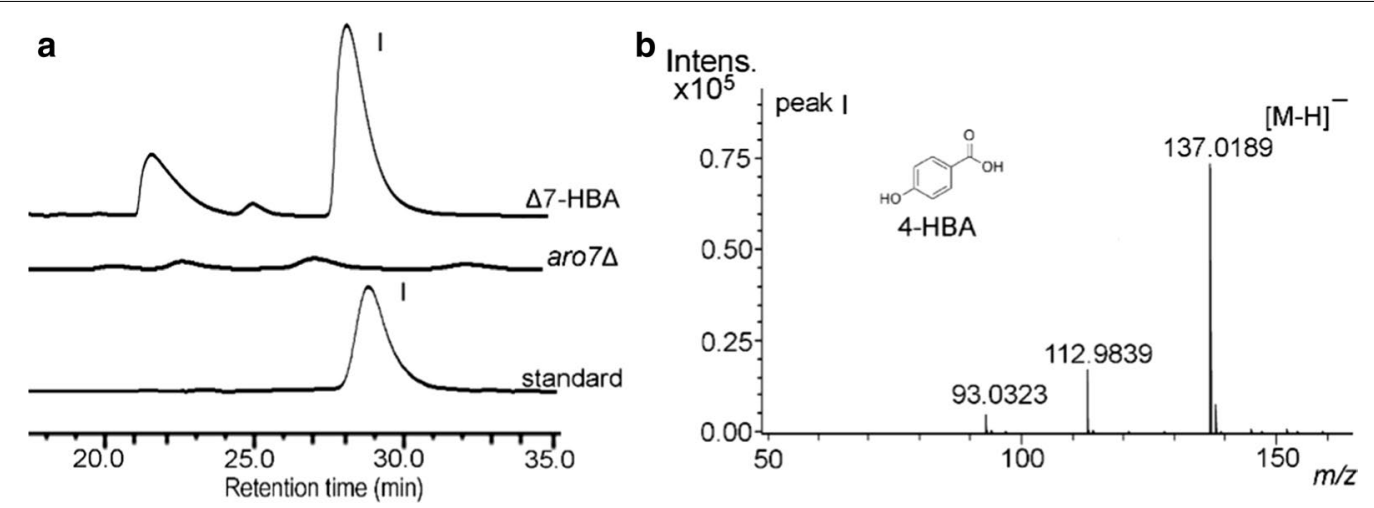

Fig. 5 The HPLC analysis and identification of 4-HBA produced in strain $\triangle 7-H B A$. a HPLC spectrum at $201 \mathrm{~nm}$ of the 4-HBA standard and fermentation supernatant of the strains S. cerevisiae aro7 $\triangle$ and $\triangle 7-H B A$ (with genes of $A R O 2, A R O 1, A R O 4^{k 229 L}, u b i C^{s y n}$, ppsA and ktA integrated into $\delta$ sites of S. cerevisiae aro $\triangle$ ). $\mathbf{b}$ Mass spectrum of peak I in the fermentation supernatant of $\triangle 7-H B A$, which was collected and analyzed by LC-MS in negative ion mode in a methanol-water (containing $20 \mathrm{mM} \mathrm{NH}_{4} \mathrm{Ac}$ ) gradient system 


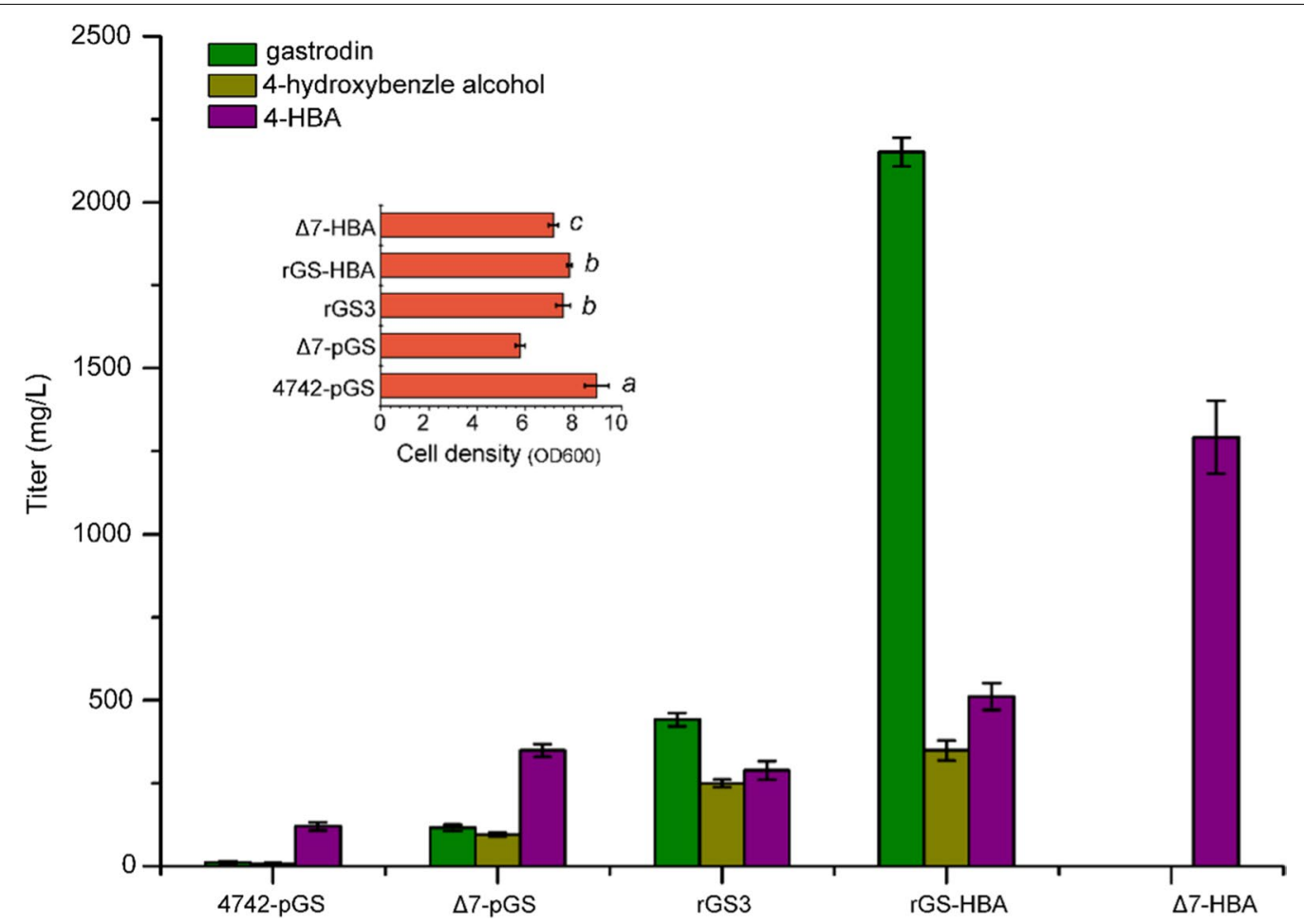

Fig. 6 Titers of gastrodin, 4-hydroxybenzyl alcohol and 4-HBA, and the cell density of engineered strains. The strain 4742-pGS, S. cerevisiae BY4742

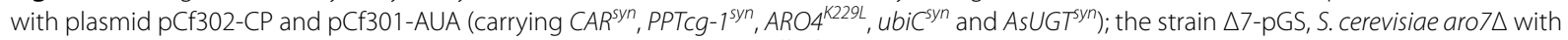

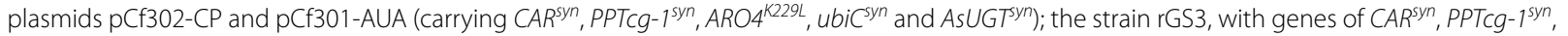

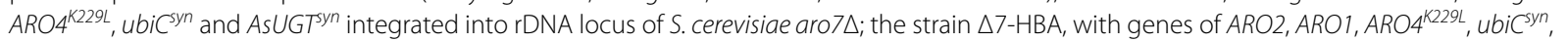
pps $A$ and ktA integrated into $\delta$ sites of $S$. cerevisiae aro $\triangle \Delta$; the strain rGS-HBA with genes of $A R O 2, A R O 1, A R O 4^{K 229 L}$, ubi $C^{S y n}$, pps $A$ and ktA integrated into $\delta$ sites of strain rGS3. Three replicates were performed, and the error bars represented standard deviation. The significance differences of cell density between the control $\triangle 7$-pGS and other engineered strain were analyzed by Student's t-test. a, $p$-value $<0.01 ; b, p$-value $<0.05 ; c, n o$ statistical significance differences

the production of all the three compounds was increased in $\triangle 7$-pGS, indicating that deletion of ARO7 activated the shikimate pathway and promoted the biosynthesis of gastrodin, 4-hydroxybenzyl alcohol and 4-HBA. The cell density of $\triangle 7$-pGS $\left(\mathrm{OD}_{600}=5.8\right)$ was lower than that of $4742-$ pGS $\left(\mathrm{OD}_{600}=9.0\right)$, probably because deletion of $A R O 7$ affected strain growth. The plasmid-free strains rGS, $\triangle 7$-HBA and rGS-HBA showed similar cell densities, higher than that of plasmid-based $\Delta 7$-pGS. In addition to gastrodin, the total amount of 4-hydroxybenzylalcohol produced by plasmid-free rGS3 was 3 times that produced by the plasmid-based strain $\Delta 7$-pGS. These results further confirmed that the genes integrating expression in yeast were superior to plasmid-based expression in gastrodin production. With the cooverexpression of ppsA, tktA, ARO1, ARO2, ARO4 $4^{K 229 L}$ and $u b i C^{s y n}$ integrated in $\delta$ sites, $1.3 \mathrm{~g} / \mathrm{L}$ 4-HBA accumulation was observed in $\triangle 7$-HBA. The enhanced pool of 4-HBA further increased the metabolic flux toward the aglycon 4-hydroxybenzyl alcohol, and consequently, high gastrodin production $(2.1 \mathrm{~g} / \mathrm{L})$ was achieved in strain
rGS-HBA, which was approximately 4.8 times that of $S$. cerevisiae rGS. With these rational engineering, the titer of gastrodin was improved 175 times higher than that of the original 4742-pGS.

The expression levels of target genes were detected between engineered strains by quantitative real-time PCR. Relative transcript levels were analyzed individually after normalization to the actin internal reference gene. Results were as shown in Additional file 1: File S1The analysis of expression levels of target genes. The changes of transcript expression levels of target genes showed good consistency with the production of gastrodin between engineered strains.

\section{Conclusions}

Here, we explored the potential of $S$. cerevisiae as the host for high-level production of gastrodin from glucose. The glucosyltransferase AsUGT was firstly identified to convert 4-hydroxybenzylalcohol to gastrodin with high catalytic efficiency in yeast. The de novo biosynthesis of gastrodin from glucose was realized by introducing the 
$A s U G T^{s y n}$ and other pathway genes into S. cerevisiae. With multiple-copy integration of the entire pathway genes in the rDNA locus and enhancement of metabolic flux toward 4-hydroxybenzyl alcohol, the best optimized strain produced $2.1 \mathrm{~g} / \mathrm{L}$ gastrodin in mineral medium with glucose as the sole carbon source. To our knowledge, this is the highest reported titer of gastrodin produced by an engineered microorganism. Instead of chemical synthesis or plants extraction, this work provides a strong basis for the biotechnological production of gastrodin at an industrial scale for pharmaceutical applications.

\section{Material and methods}

\section{Strains and medium}

All plasmids and strains used in this work are shown in Table 1. E. coli DH5 $\alpha$ was used for plasmid construction. E. coli strains were cultivated in Luria broth (LB). S. cerevisiae BY4742 (MATa his $3 \Delta 1$ leu $2 \Delta 0$ lys $2 \Delta 0$ ura $3 \Delta 0$ ) was used as the parental strain for all the yeast strains. Yeast strains were grown in SC dropout medium containing $6.7 \mathrm{~g} / \mathrm{L}$ yeast nitrogen base, $2 \mathrm{~g} / \mathrm{L}$ dropout mix, and $20 \mathrm{~g} / \mathrm{L}$ glucose. SC dropout medium without uracil (SCura), SC dropout medium without leucine (SC-leu), and
SC dropout medium without uracil and leucine (SC-ura, leu) were used in this work. Yeast strains were also cultured in YPD medium containing $10 \mathrm{~g} / \mathrm{L}$ yeast extract, $20 \mathrm{~g} / \mathrm{L}$ peptone and $20 \mathrm{~g} / \mathrm{L}$ glucose. Standards, including gastrodin, 4-hydroxybenzl alcohol and 4-HBA, were purchased from Aladdin Chemistry Co., Ltd. (Shanghai, China).

\section{Genes and enzymes}

Glucosyltransferase AsUGT (Q9AR73) [17] from Rauvolfia serpentina (serpentwood), Rhodiola-derived glycosyltransferase UGT73B6 mutant gene $U G T 73 B 6^{F S}$ [11], carboxylic acid reductase CAR (Q6RKB1.1) gene [21] from Nocardia iowensis, phosphopantetheinyl transferase PPTcg-1 (WP_003857486.1) gene [21] from Corynebacterium glutamicum, and chorismate pyruvate-lyase UbiC (AAY88959.1) [34] gene from E. coli BL21 (DE3) were synthesized with $S$. cerevisiae codon optimization by Generay Biotech Co., Ltd. (Shanghai, China). The feedback-resistant DAHP synthase mutant $\mathrm{ARO} 4^{\mathrm{K} 229 \mathrm{~L}}$ from S. cerevisiae was generated from DAHP synthase ARO4 (NP_009808) by a site-directed mutagenesis method. The pentafunctional EPSP synthase gene ARO1 (Gene ID:

Table 1 Strains and plasmids used in this study

\begin{tabular}{|c|c|c|}
\hline & Description & Reference \\
\hline \multicolumn{3}{|l|}{ Strains } \\
\hline S. cerevisiae BY4742 & MATa; his3 $\Delta 1$; leu2 $\triangle 0$; lys $2 \Delta 0$; ura3 $\Delta 0$ & Novagen \\
\hline $4742-301$ & BY4742 with pCf301 & This study \\
\hline 4742-UGT73B6 $6^{\text {FSy }}$ & BY4742 with pCf301-UGT73B6 $6^{F S y}$ & This study \\
\hline $4742-A s U G T^{\text {syn }}$ & BY4742 with pCf301-AsUGT $T^{\text {syn }}$ & This study \\
\hline 4742-pCf & BY4742 with pCf301\&pCf302 & This study \\
\hline 4742-pGS & BY4742 with pCf302-CP \& pCf301-AUA & This study \\
\hline S. cerevisiae aro7 $\triangle$ & The deletion of ARO7 in S. cerevisiae BY4742 & This study \\
\hline$\triangle$ aro7-pCf & S. cerevisiae aro7D with pCf301 \& pCf302 & This study \\
\hline$\Delta 7-p G S$ & S. cerevisiae aro7 $\triangle$ with pCf302-CP \& pCf301-AUA & This study \\
\hline rGS3 & 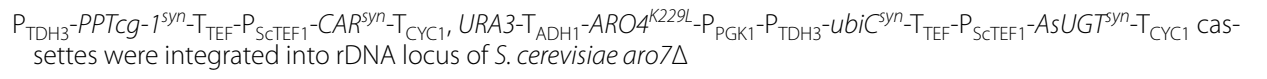 & This study \\
\hline$\triangle 7-H B A$ & 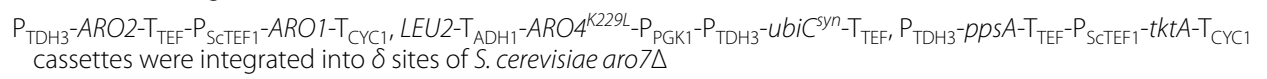 & This study \\
\hline rGS-HBA & 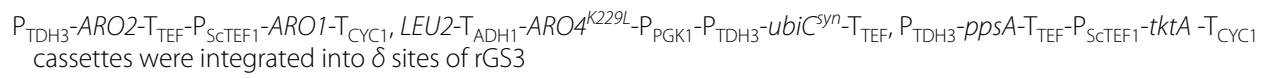 & This study \\
\hline \multicolumn{3}{|c|}{ 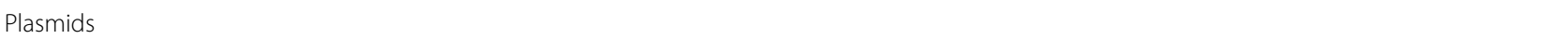 } \\
\hline pCf301 & URA3; $2 \mu \mathrm{m}$ ori with $\mathrm{P}_{\mathrm{PGK} 1}, \mathrm{P}_{\mathrm{TDH}}, \mathrm{P}_{\mathrm{TEF} 1} ; A m p$ & In our lab 30 \\
\hline pCf302 & LEU2; $2 \mu m$ ori with $\mathrm{P}_{\mathrm{PGK} 1}, \mathrm{P}_{\mathrm{TDH} 3}, \mathrm{P}_{\mathrm{TEF} 1} ; A m p$ & In our lab 30 \\
\hline pCf301-UGT73B6 F5y & pCf301 carrying $P_{\text {SCTEF1 }}-U G T 73 B 6^{F S y}-T_{C Y C 1}$ & This study \\
\hline pCf301-AsUGT $T^{\text {yn }}$ & pCf301 carrying $P_{\text {SCTEF1 }}-$ AsUGT $^{\text {Syn }}-T_{C Y C 1}$ & This study \\
\hline $\mathrm{pCf302-CP}$ & pCf302 carrying $\mathrm{P}_{\mathrm{SCTEF} 1}-C A R^{\text {Syn }}-\mathrm{T}_{\mathrm{CYC} 1}, \mathrm{P}_{\mathrm{TDH} 3}-\mathrm{PPTCg}_{-1} 1^{\mathrm{Syn}}-\mathrm{T}_{\mathrm{TEF}}$ & This study \\
\hline pCf301-AUA & 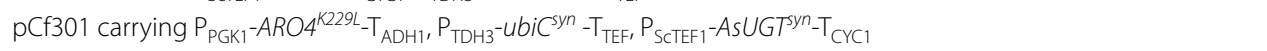 & This study \\
\hline pCf302-AU & pCf302 carrying $\mathrm{P}_{\mathrm{PGK} 1}-A R O 4^{K 229 L}-\mathrm{T}_{\mathrm{ADH} 1}, \mathrm{P}_{\mathrm{TDH}}-\mathrm{Ubi}^{\mathrm{Syn}}-\mathrm{T}_{\mathrm{TEF}}$ & This study \\
\hline pCf301-pt & pCf301 carrying $P_{\mathrm{TDH}}-$ pps $-T_{\mathrm{TEF}}-\mathrm{P}_{\mathrm{SCTEF} 1}-t k t A-T_{\mathrm{CYC} 1}$ & This study \\
\hline $\mathrm{pCf} 301-A R 01 / 2$ & $\mathrm{pCf} 301$ carrying $\mathrm{P}_{\mathrm{TDH} 3}-A R O-\mathrm{T}_{\mathrm{TEF}}-\mathrm{P}_{\mathrm{SCTEF} 1}-A R O 1-\mathrm{T}_{\mathrm{CYC} 1}$ & This study \\
\hline
\end{tabular}


851705) and chorismate synthase gene ARO2 (GeneID: 852729) were amplified from $S$. cerevisiae BY4742. The phosphoenolpyruvate synthase gene ppsA (Gene ID: 946209) and transketolase gene $t k t A$ (Gene ID: 947420) were amplified from $E$. coli MG1655. The synthesized genes and sequences applied in this study are showed in Additional file 1: Table S2.

\section{Vector construction}

All the primers used in this study are listed in Additional file 1: Table S1.

The codon-optimized genes AsUGT $T^{\text {syn }}$ and UGT$73 B 6^{F S y}$ in $S$. cerevisiae were separately cloned into the pCf301 [30] vector using NheI/AatII to create the plasmids pCf301- AsUGT ${ }^{\text {syn }}$ and pCf301- UGT73B6 $6^{F S y}$, respectively. The codon-optimized genes $C A R^{s y n}$ and $P P T c g-1^{\text {syn }}$ were respectively cloned into the pCf302 [30] vector using NheI/AatII and HindIII/BamHI to create the pCf302-CP plasmids. The ARO4 ${ }^{K 229 L}$, codon-optimized $A s U G T^{s y n}$, and $u b i C^{s y n}$ genes were respectively cloned into the pCf301 vector using SpeI/BglII, NheI/AatII and HindIII/BamHI to create the pCf301-AUA plasmids. The genes $A R O 4^{K 229 L}$ and $u b i C^{s y n}$ were also respectively cloned into the pCf302 vector using SpeI/BglII and Hin$d \mathrm{III} / \mathrm{BamHI}$ to create the $\mathrm{pCf} 302-\mathrm{AU}$ plasmids. The $p p s A$ and $t k t A$ genes were respectively cloned into the pCf301 vector using NheI/AatII and HindIII/BamHI to create the pCf301-pt plasmids. The genes $A R O 1$ and $A R O 2$ were respectively cloned into the pCf301 vector using NheI/ AatII and HindIII/BamHI to create the pCf301-ARO1/2 plasmids.

\section{Chromosomal disruption of $A R O 7$}

Targeted chromosomal disruption of the chorismate mutase gene ARO7 in the strain S. cerevisiae BY4742 was performed by homologous recombination using antibiotic marker-containing disruption cassettes created by PCR, as described by Gueldener et al. [35]. The loxPflanked $\operatorname{kanMX}$ cassettes were amplified from pUG6. The antibiotic marker was removed using the Cre recombinase plasmid pSH65. Clones successfully carrying the ARO7 disruption cassette were further confirmed by colony PCR and named S. cerevisiae BY4742 aro7A.

\section{Feeding experiments}

The $S$. cerevisiae BY4742 strains respectively harboring pCf301, pCf301-UGT73B6 ${ }^{F S y}$ or pCf301-AsUGT ${ }^{\text {syn }}$ were cultured in $3 \mathrm{~mL}$ of SC-ura medium at $30^{\circ} \mathrm{C}$ and 220 r.p.m. for $24 \mathrm{~h}$. Then, $2 \mathrm{~mL}$ of the culture was diluted into $50 \mathrm{~mL}$ of medium and shaken at $30{ }^{\circ} \mathrm{C}$ at 220 r.p.m. for $18 \mathrm{~h}$. Then, the substrate 4-hydroxybenzyl alcohol was added into the cultures at a final concentration of $2 \mathrm{mM}$. After $24 \mathrm{~h}$ and $48 \mathrm{~h}$, samples were taken and analyzed by
HPLC or HPLC-MS. Three replicates were performed in experiments. The data were presented as means $\pm S D$ (standard deviation).

\section{Yeast cultivation}

All measurements of optical density at $600 \mathrm{~nm}\left(\mathrm{OD}_{600}\right)$ were made using a SHIMADZU UV-1800 spectrophotometer. A single colony was first inoculated into $35-\mathrm{mL}$ culture tubes containing $3 \mathrm{~mL}$ of SC dropout medium and grown at $30{ }^{\circ} \mathrm{C}$ and 220 r.p.m. for $18 \mathrm{~h}$. Then, seed cultures were transferred into $50 \mathrm{~mL}$ of SC dropout medium to obtain an $\mathrm{OD}_{600}$ of 0.2 in $250-\mathrm{mL}$ culture flasks. The strains were cultured at $30{ }^{\circ} \mathrm{C}$ and 220 r.p.m. for approximately 7 days. Samples were taken every $24 \mathrm{~h}$ and analyzed by HPLC. All of the data generated in this study were collected from three independent experiments. The data were presented as means $\pm S D$ (standard deviation).

\section{One-step multiple-fragment yeast transformation}

The procedure of multiple-fragment transformation was based on the method described by Jolanda van Leeuwen for rapid and efficient plasmid construction [36]. A single colony was inoculated into $3 \mathrm{~mL}$ of liquid medium and incubated overnight at 200 r.p.m. and $30{ }^{\circ} \mathrm{C}$. Then, $2 \mathrm{~mL}$ of the overnight culture was diluted into $50 \mathrm{~mL}$ of medium to obtain an $\mathrm{OD}_{600}$ of 0.2 and incubated at $30{ }^{\circ} \mathrm{C}$ and 200 r.p.m. The culture with an $\mathrm{OD}_{600}$ of 0.8 was centrifuged at $2000 \mathrm{~g}$ for $3 \mathrm{~min}$, and the cell pellet was suspended in $25 \mathrm{~mL}$ of sterile water. After centrifugation, the cells were suspended in $1.0 \mathrm{~mL}$ of $0.1 \mathrm{M}$ lithium acetate (LiAc). The cell suspension was transferred to a $1.5-\mathrm{mL}$ microcentrifuge tube and centrifuged for $15 \mathrm{~s}$ at $2000 \mathrm{~g}$. After discarding the supernatant, the cells were suspended in $320 \mu \mathrm{L}$ of $\mathrm{LiAc}(0.1 \mathrm{M})$ as competent cells. Ten microliters of DNA fragment mixture (100-200 ng of each DNA fragment), $2 \mu \mathrm{L}$ of ssDNA $(10 \mathrm{mg} / \mathrm{mL})$ and $12 \mu \mathrm{L}$ of competent yeast cells were added into a $1.5-\mathrm{mL}$ microcentrifuge tube and mixed gently. Then, $100 \mu \mathrm{L}$ of the transformation buffer $(800 \mu \mathrm{L}$ of $50 \%$ PEG3350, $100 \mu \mathrm{L}$ of $1 \mathrm{M} \mathrm{LiAc}, 100 \mu \mathrm{L}$ of $10 \times \mathrm{TE}$, and $50 \mu \mathrm{L}$ of DMSO) was added into the $1.5-\mathrm{mL}$ microcentrifuge tube and vortexed for $10 \mathrm{~s}$. After incubation for $30 \mathrm{~min}$ at $25^{\circ} \mathrm{C}$, the transformation mixture was incubated at $42^{\circ} \mathrm{C}$ for $15 \mathrm{~min}$ and then on ice for $5 \mathrm{~min}$. The transformation mixture was centrifuged at $800 \mathrm{~g}$ for $1 \mathrm{~min}$, and the cells were suspended in $1 \mathrm{~mL}$ of YPD. The cell suspension was incubated at $30^{\circ} \mathrm{C}$ for $2 \mathrm{~h}$ and was centrifuged at $2000 \mathrm{~g}$ for $1 \mathrm{~min}$. The cells were suspended in sterile water and plated on SC plates lacking leucine and/or uracil. Plasmid transformation was carried out using the standard LiAc method. 


\section{Chromosomal multiple-copy integration of the pathway genes for gastrodin biosynthesis}

The $C A R^{s y n}, \quad P P T c g-1^{s y n}, A s U G T^{s y n}, u b i C^{s y n}$ and $A R O 4^{K 229 L}$, URA3 selectable marker, a total sequence of approximately $14 \mathrm{~kb}$, were integrated into the ribosomal DNA (rDNA) locus. The two fragments rDNA-up (476 bp) and rDNA-down (326 bp) were PCR-amplified from the DNA of $S$. cerevisiae BY4742 using the primer set $r U-F \& R$ and $r D-F \& R$. An approximately 5.8-kb fragment containing $C A R^{s y n}$ and $P P T c g-1^{\text {syn }}$ was PCR-amplified from the pCf302-CP plasmids using the primer set $\mathrm{r} 5.8-\mathrm{F} \& \mathrm{R}$. An approximately 7.4-kb fragment containing AsUGT ${ }^{s y n}, u b i C^{s y n}, A R O 4^{K 229 L}$ and URA3 was PCR-amplified from the pCf301-AUA plasmids using the primer set r7.4-F \& R. The four DNA fragments, with 30-40 bp homologous regions designed between the adjacent fragments, were transferred into S. cerevisiae aro7A using the protocol for one-step multiple-fragment transformation mentioned above. The colonies were selected on the $\mathrm{SC}$-ura plates and verified by PCR amplification and sequencing. Positive colonies were cultured, and the highest gastrodinproducing strain was designated rGS3.

The ARO1, ARO2, ppsA, tktA, ubiC ${ }^{s y n}, A R O 4^{K 229 L}$ and LEU2 selectable marker, a total sequence length of approximately $21 \mathrm{~kb}$, were integrated separately into the $\delta$-sites of the strains $S$. cerevisiae aro7 $\Delta$ and rGS3. The two fragments $\delta$ DNA-up (329 bp) and $\delta$ DNAdown (316 bp) were PCR-amplified from the DNA of $S$. cerevisiae BY4742 using the primer set $\delta$ U-F\&R and $\delta$ D-F\&R. A 7.7-kb fragment containing ARO1 and ARO2 was PCR-amplified from the pCf301-ARO1/2 plasmid using the primer set $\delta 7.7-F \& R$. An approximately 6.8$\mathrm{kb}$ fragment containing $\mathrm{LEU} 2, \mathrm{ubiC}^{\mathrm{Syn}}$, and $\mathrm{ARO} 4^{\mathrm{K} 229 \mathrm{~L}}$ was PCR-amplified from the pCf302-AU plasmid using the primer set $\delta 6.8$-F\&R. A $6.2-\mathrm{kb}$ fragment containing ppsA and $t k t A$ was amplified from the pCf301-pt plasmid using the primer set $\delta 6.2-\mathrm{F} \& \mathrm{R}$. The five DNA fragments, with 30-40 bp homologous regions designed between the adjacent fragments, were transformed into the strain S. cerevisiae aro7 $\Delta$ using the protocol for one-step multiple-fragment transformation mentioned above. Positive colonies were selected on the $\mathrm{SC}$-leu plates, and the strain with the highest 4-HBA production was designated strain $\triangle 7$-HBA. The five DNA fragments were also transformed into the strain rGS3. Colonies were selected on the SC-ura, leu plates, and the strain with the highest gastrodin production was designated strain rGS-HBA. The sequences of the rDNA and $\delta$ DNA fragments applied are shown in Additional file 1: Table S2.

\section{Metabolite analysis}

The chemicals gastrodin, 4-hydroxybenzl alcohol and 4-HBA were analyzed by HPLC on a Shimadzu system with a UV detector. The column used was an Agela Innoval C18, $4.6 \times 250 \mathrm{~mm}$, with a particle size of $5 \mu \mathrm{m}$. A methanol-water (containing $0.1 \%$ trifluoroacetic acid) gradient system at a flow rate of $1 \mathrm{~mL} / \mathrm{min}$ was used to separate the compounds. The HPLC program was as follows: $10 \%$ methanol for $40 \mathrm{~min}, 10 \%$ to $100 \%$ methanol for $1 \mathrm{~min}, 100 \%$ methanol for $10 \mathrm{~min}, 100 \%$ to $10 \%$ methanol for $1 \mathrm{~min}$, and $10 \%$ methanol for $10 \mathrm{~min}$. Products were detected at $224 \mathrm{~nm}$ or $201 \mathrm{~nm}$. HPLC-MS was performed on an Agilent 1260 system with a 1260 Infinity UV detector and a Bruker micro Q-TOF II mass spectrometer equipped with an ESI ionization probe. The HPLC conditions were the same as described above. For HPLC-MS analysis of 4-HBA, the fraction of the peak was collected for MS identification in negative ion mode, and methanol-water (containing $20 \mathrm{mM} \mathrm{NH}_{4} \mathrm{Ac}$ ) was used as the gradient system. The other conditions were the same as described above. To quantify the titers of gastrodin, 4-hydroxybenzl alcohol and 4-HBA, standard calibration curves were prepared with a series of known concentrations of the standard. R2 value for the standard curve was higher than 0.999. Samples were diluted as required for falling into the concentration range of the calibration curves. The titers were presented as means \pm SD (standard deviation).

\section{RNA isolation and quantitative real-time PCR}

Total RNA from S. cerevisiae was isolated and purified using hot-acid-phenol method. To synthesize cDNA, about $2.0 \mu \mathrm{g}$ of RNA was added to a FastKing gDNA Dispelling RT SuperMix (TIANGEN Biotech, Beijing, China). Each $20.0 \mu \mathrm{L}$ reaction contained $10.0 \mu \mathrm{L}$ of SuperReal PreMix Plus (SYBR green) (TIANGEN Biotech, Beijing, China). Real-time PCR was monitored and analyzed using Roche LightCycler ${ }^{\circledR} 96$ System. Samples of $\Delta 7$-pGS and rGS3 were prepared after 2 days and 4 days fermentation culture. Samples of rGS3-PHB were prepared after 2 days. All samples were prepared in triplicate. The relative expression level of genes in engineered strain was calculated by the relative quantitative $2^{-\Delta \Delta C T}$ method [37] and B-actin was used as the internal control gene Additional file 1: File S1.

\section{Supplementary information}

Supplementary information accompanies this paper at https://doi. org/10.1186/s12934-020-01476-0. 
Additional files. File S1. The analysis of expression levels of target genes. Figure S1. Titer of gastrodin produced by colonies with gene integration into the yeast chromosomal rDNA locus. Figure S2. Titer of 4-HBA accumulated in the culture of colonies with gene integration into yeast chromosomal $\delta$ sites. Figure $\mathbf{S 3}$. Titer of gastrodin produced by colonies with gene integration into yeast chromosomal $\delta$ sites. Figure $\mathbf{S} 4$. The relative transcript levels of target genes in rGS3 compared to $\triangle 7$-pGS at 2 day and 4 day. Figure $\mathbf{S 5}$. The relative transcript levels of target genes in rGS3-PHB compared to rGS3 at 2 day. Table S1. The primers used in this study. Table S2. The synthesized genes, sequence of rDNA and $\delta$ DNA fragment applied in this study. Table S3. The real-time PCR primers used in this study. Table S4. Information of genes used in this study.

\section{Acknowledgements}

This work was supported by grants from the National Natural Science Foundation of China (31400026, 31970065, U1902214), the Yunnan Key Research and Development Program (2019ZF011-2), and the Tianjin Synthetic Biotechnology Innovation Capacity Improvement Project (TSBICIP-KJGG-002). We thank Jie Zhang at Laboratory of Structural Biology (TIB, CAS) for technical help with RT-PCR experiments.

\section{Authors' contributions}

$\mathrm{TL}$ and $\mathrm{HY}$ conceived the project, analyzed results, and wrote the manuscript. HY performed most of the experiments. HY conducted gastrodin fermentation with contribution from TH and YZ. All authors commented on the manuscript. All authors read and approved the final manuscript

\section{Availability of data and materials}

All data generated or analyzed during this study are included in this article and its Additional file 1.

\section{Ethics approval and consent to participate} Not applicable.

\section{Consent for publication}

Not applicable.

\section{Competing interests}

Part of the work has been included in a patent application by the Tianjin Institute of Industrial Biotechnology.

\section{Author details}

${ }^{1}$ Tianjin Institute of Industrial Biotechnology, Chinese Academy of Sciences, Tianjin 300308, China. ${ }^{2}$ Key Laboratory of Systems Microbial Biotechnology, Chinese Academy of Sciences, Tianjin 300308, China.

Received: 8 August 2020 Accepted: 13 November 2020

Published online: 26 November 2020

\section{References}

1. Matias M, Silvestre S, Falcao A, Alves G. Gastrodia elata and epilepsy: Rationale and therapeutic potential. Phytomedicine. 2016;23:1511-26.

2. Chinese Pharmacopoeia Commission. Pharmacopoeia of the People's Republic of China. Chinese Medical Science and Technology Press (Beijing). 2010; 54-55.

3. Liu Y, Gao J, Peng M, Meng H, Ma H, Cai P, Xu Y, Zhao Q, Si G. A review on central nervous system effects of gastrodin. Front Pharmacol. 2018;9:24.

4. Deng CK, Mu ZH, Miao YH, Liu YD, Zhou L, Huang YJ, Zhang F, Wang YY, Yang ZH, Qian ZY, et al. Gastrodin ameliorates motor learning deficits through preserving cerebellar long-term depression pathways in diabetic rats. Front Neurosci. 2019;13:1239.

5. Jiang T, Chu J, Chen H, Cheng H, Su J, Wang X, Cao Y, Tian S, Li Q. Gastrodin Inhibits H2O2-Induced Ferroptosis through Its Antioxidative Effect in Rat Glioma Cell Line C6. Biol Pharm Bull. 2020;43:480-7.

6. Peng Z, Wang S, Chen G, Cai M, Liu R, Deng J, Liu J, Zhang T, Tan Q, Hai C. Gastrodin alleviates cerebral ischemic damage in mice by improving anti-oxidant and anti-inflammation activities and inhibiting apoptosis pathway. Neurochem Res. 2015;40:661-73.

7. Hu M, Yan H, Fu Y, Jiang Y, Yao W, Yu S, Zhang L, Wu Q, Ding A, Shan M. Optimal Extraction Study of Gastrodin-Type Components from Gastrodia Elata Tubers by Response Surface Design with Integrated Phytochemical and Bioactivity Evaluation. Molecules. 2019;24:547.

8. Liu Y, Huang G. The content analysis of gastrodin and gastrodigenin obtained by different processing methods. J Chromatogr Sci. 2018:56:65-7.

9. Tsai CC, Wu KM, Chiang TY, Huang CY, Chou CH, Li SJ, Chiang YC. Comparative transcriptome analysis of Gastrodia elata (Orchidaceae) in response to fungus symbiosis to identify gastrodin biosynthesis-related genes. BMC Genomics. 2016;17:212.

10. Borodina I, Nielsen J. Advances in metabolic engineering of yeast Saccharomyces cerevisiae for production of chemicals. Biotechnol J. 2014;9:609-20.

11. Bai Y, Yin H, Bi H, Zhuang Y, Liu T, Ma Y. De novo biosynthesis of Gastrodin in Escherichia coli. Metab Eng. 2016;35:138-47.

12. Cao M, Gao M, Suastegui M, Mei Y, Shao Z. Building microbial factories for the production of aromatic amino acid pathway derivatives: from commodity chemicals to plant-sourced natural products. Metab Eng. 2020;58:94-132.

13. Gao S, Lyu Y, Zeng W, Du G, Zhou J, Chen J. Efficient Biosynthesis of (2S)Naringenin from p-Coumaric Acid in Saccharomyces cerevisiae. J Agric Food Chem. 2020;68:1015-21.

14. Averesch NJH, Kromer JO. Metabolic engineering of the shikimate pathway for production of aromatics and derived compounds-present and future strain construction strategies. Front Bioeng Biotechnol. 2018;6:32.

15. Liu Q, Yu T, Li X, Chen Y, Campbell K, Nielsen J, Chen Y. Rewiring carbon metabolism in yeast for high level production of aromatic chemicals. Nat Commun. 2019;10:4976.

16. Nielsen J. Yeast systems biology. Model organism and cell factory. Biotechnol J. 2019;14:e1800421.

17. Hefner T, Arend J, Warzecha H, Siems K, Stockigt J. Arbutin synthase, a novel member of the NRD1 beta glycosyltransferase family, is a unique multifunctional enzyme converting various natural products and xenobiotics. Bioorg Med Chem. 2002;10:1731-41.

18. Winter G, Averesch NJ, Nunez-Bernal D, Kromer JO. In vivo instability of chorismate causes substrate loss during fermentative production of aromatics. Yeast. 2014;31:333-41.

19. Marbois B, Xie LX, Choi S, Hirano K, Hyman K, Clarke CF. para-Aminobenzoic acid is a precursor in coenzyme Q6 biosynthesis in Saccharomyces cerevisiae. J Biol Chem. 2010;285:27827-38.

20. Kromer JO, Nunez-Bernal D, Averesch NJ, Hampe J, Varela J, Varela C. Production of aromatics in Saccharomyces cerevisiae-a feasibility study. J Biotechnol. 2013;163:184-93.

21. Hansen EH, Moller BL, Kock GR, Bunner CM, Kristensen C, Jensen OR, Okkels FT, Olsen CE, Motawia MS, Hansen J. De novo biosynthesis of vanillin in fission yeast (Schizosaccharomyces pombe) and baker's yeast (Saccharomyces cerevisiae). Appl Environ Microbiol. 2009;75:2765-74.

22. Romanos MA, Scorer CA, Clare JJ. Foreign gene expression in yeast: a review. Yeast. 1992;8:423-88.

23. Erhart $E$, Hollenberg CP. The presence of a defective LEU2 gene on $2 \mathrm{mu}$ DNA recombinant plasmids of Saccharomyces cerevisiae is responsible for curing and high copy number. J Bacteriol. 1983;156:625-35.

24. Englaender JA, Jones JA, Cress BF, Kuhlman TE, Linhardt RJ, Koffas MAG. Effect of genomic integration location on heterologous protein expression and metabolic engineering in E. coli. ACS Synth Biol. 2017;6:710-20.

25. Lv Y, Edwards H, Zhou J, Xu P. Combining 26s rDNA and the Cre-loxP System for Iterative Gene Integration and Efficient Marker Curation in Yarrowia lipolytica. ACS Synth Biol. 2019;8:568-76.

26. Wery J, Gutker D, Renniers AC, Verdoes JC, van Ooyen AJ. High copy number integration into the ribosomal DNA of the yeast Phaffia rhodozyma. Gene. 1997;184:89-97.

27. Parekh RN, Shaw MR, Wittrup KD. An integrating vector for tunable, high copy, stable integration into the dispersed Ty delta sites of Saccharomyces cerevisiae. Biotechnol Prog. 1996;12:16-21.

28. Dai Z, Liu Y, Zhang X, Shi M, Wang B, Wang D, Huang L, Zhang X. Metabolic engineering of Saccharomyces cerevisiae for production of ginsenosides. Metab Eng. 2013;20C:146-56. 
29. Wang C, Su X, Sun M, Zhang M, Wu J, Xing J, Wang Y, Xue J, Liu X, Sun W, Chen S. Efficient production of glycyrrhetinic acid in metabolically engineered Saccharomyces cerevisiae via an integrated strategy. Microb Cell Fact. 2019;18:95.

30. Jiang JJ, Yin H, Wang SA, Zhuang YB, Liu SW, Liu T, Ma YH. Metabolic Engineering of Saccharomyces cerevisiae for High-Level Production of Salidroside from Glucose. J Agric Food Chem. 2018:66:4431-8.

31. Suastegui M, Guo W, Feng X, Shao Z. Investigating strain dependency in the production of aromatic compounds in Saccharomyces cerevisiae. Biotechnol Bioeng. 2016;113:2676-85.

32. Gao M, Cao M, Suastegui M, Walker J, Rodriguez Quiroz N, Wu Y, Tribby D, Okerlund A, Stanley L, Shanks JV, Shao Z. Innovating a nonconventional yeast platform for producing shikimate as the building block of highvalue aromatics. ACS Synth Biol. 2017:6:29-38.

33. Koma D, Kishida T, Yoshida E, Ohashi H, Yamanaka H, Moriyoshi K, Nagamori E, Ohmoto T. Chromosome engineering to generate plasmid-free phenylalanine- and tyrosine-overproducing Escherichia coli which can be applied in generation of aromatic compound-producing strains. Appl Environ Microbiol. 2020. https://doi.org/10.1128/AEM.00525-20.
34. Siebert M, Severin K, Heide L. Formation of 4-Hydroxybenzoate in Escherichia Coli Characterization of the Ubic Gene and Its Encoded Enzyme Chorismate Pyruvate-Lyase. Microbiology-Sgm. 1994;140:897-904.

35. Gueldener U, Heinisch J, Koehler GJ, Voss D, Hegemann JH. A second set of loxP marker cassettes for Cre-mediated multiple gene knockouts in budding yeast. Nucleic Acids Res. 2002;30:e23.

36. Van Leeuwen J, Andrews B, Boone C, Tan G. Rapid and efficient plasmid construction by homologous recombination in yeast. Cold Spring Harb Protoc. 2015;2015:pdb prot085100.

37. Schmittgen TD, Livak KJ. Analyzing real-time PCR data by the comparative $C_{T}$ method. Nat Protoc. 2008;3:1101-8.

\section{Publisher's Note}

Springer Nature remains neutral with regard to jurisdictional claims in published maps and institutional affiliations.
Ready to submit your research? Choose BMC and benefit from:

- fast, convenient online submission

- thorough peer review by experienced researchers in your field

- rapid publication on acceptance

- support for research data, including large and complex data types

- gold Open Access which fosters wider collaboration and increased citations

- maximum visibility for your research: over $100 \mathrm{M}$ website views per year

At BMC, research is always in progress.

Learn more biomedcentral.com/submissions 